\title{
Thermal nonlinearity in silicon microcylindrical resonators
}

Natasha Vukovic, ${ }^{1}$, a) Noel Healy, ${ }^{1}$ Priyanth Mehta, ${ }^{1}$ Todd D. Day, ${ }^{2}$ Pier J. A. Sazio, ${ }^{1}$

John V. Badding, ${ }^{2}$ and Anna C. Peacock ${ }^{1}$

1) Optoelectronics Research Centre, University of Southampton, Southampton SO17 1BJ, UK

2) Department of Chemistry and Materials Research Institute, Pennsylvania State University, 16802 PA, USA

(Dated: 13 April 2012)

We explore the thermally induced nonlinearity in hydrogenated amorphous silicon microcylindrical resonators that are fabricated from the silicon optical fiber platform. In particular, we use a pump-probe technique to experimentally demonstrate thermally induced optical modulation and determine the response time. Through characterization of the thermal properties and the associated resonance wavelength shifts we will show that it is possible to infer the material absorption coefficient for a range of whispering gallery mode resonators.

\footnotetext{
a)Electronic mail: ntv@orc.soton.ac.uk
} 
Whispering gallery mode (WGM) resonators which allow for investigations of light confinement in a range of geometries and materials are an area of growing research interest owing to their diverse application base. In particular, their small mode volumes and high quality factors $(Q)$ provide a convenient tool to study a variety of nonlinear phenomena including lasing, parametric amplification, and Raman scattering ${ }^{1-3}$. It is evident that of the nonlinear effects, the thermal nonlinearity has the lowest threshold so that it can be observed at powers as low as tens of microwatts. However, these thermal effects suffer from a slow response time which is typically of the order of microseconds ${ }^{3}$, limiting device performance. Nevertheless, a range of thermally induced phenomena have been observed including bistability $^{4}$ and oscillatory instability ${ }^{5}$.

Motivated by the current activities in silicon photonics, thermally induced optical bistability in WGM resonators fabricated from the silicon-on-insulator (SOI) technology have been used to demonstrate all-optical functionalities such as switching, modulation, and memory devices $^{3}$. These on-chip resonators are defined using standard photolithographic processes before being etched from the substrate, which typically imposes a nanometer scale roughness to their surfaces. As an alternative, we recently proposed an approach to fabricating silicon WGM resonators from the silicon fiber platform ${ }^{6}$. An important advantage of this method is that the resonator surfaces are defined by the ultra smooth silica cladding template ${ }^{7}$, so that the surface scattering losses are negligible compared to the chip-based resonators. Furthermore, it is straightforward to fabricate resonators of various sizes from a range of semiconductor materials that can be incorporated into the fiber geometry using simple and inexpensive processing techniques ${ }^{8,9}$.

In this paper we explore the thermally induced nonlinear effects in hydrogenated amorphous silicon (a-Si:H) fiber based microresonators. Owing to the large thermo-optic effect of a-Si:H, there is a measurable increase in the refractive index as the resonator temperature is raised through light absorption, which results in a red shift of the WGM resonances. By exploiting the power dependence of this shift, the thermal nonlinearity can be used as an efficient means to induce modulation. In our analysis we use a simple modulation experiment to characterize the thermal response of the fiber based microresonators and show that this can be used to infer the absorption coefficient of the a-Si:H material. This loss characterization method is non-destructive, localized to the point of measurement, and potentially applicable to a variety of geometries including spherical shaped semiconductor WGM resonators ${ }^{9}$. 
The silicon resonators used in our investigations are fabricated using a two-step process. Firstly, the silicon core fibers are produced by infiltrating the semiconductor material into silica capillaries with a pore diameter of $5.6 \mu \mathrm{m}$ using a high pressure microfluidic chemical deposition technique ${ }^{10}$. The silica template is then completely etched away from the a-Si:H core using a buffered HF solution to reveal the microcylindrical resonator ${ }^{6}$. Significantly, the losses of the a-Si:H resonator materials can be established through optical transmission experiments of the fibers before the cladding is etched away to form the resonators. Using the standard cutback method we have measured the losses in three silicon fibres, all fabricated to have slightly different a-Si:H material qualities so that the corresponding values are: (A) $2.5 \mathrm{~dB} / \mathrm{cm}$, (B) $6 \mathrm{~dB} / \mathrm{cm}$ and (C) $14 \mathrm{~dB} / \mathrm{cm}$ at an input wavelength of $1.55 \mu \mathrm{m}$. The difference in the losses of the three fibers is attributed to variations in the concentration of the incorporated hydrogen, which thus determines the degree of saturation of dangling bonds in the material ${ }^{10}$.

The optical properties of the microresonators are characterized over an extended telecoms band using evanescent coupling from a tapered single mode fiber with a waist diameter of $\sim 1-2 \mu \mathrm{m}$. A polarization controller was used before the taper to adjust the polarization state of the fiber taper mode, and to provide selective coupling to the TE or TM WGMs of the cylindrical resonators. A typical transmission spectrum of the low loss fiber (A) is shown in Fig. 1(a) illustrating the sharp resonances associated with the WGMs of the resonator. Two individual resonances are highlighted, $\lambda_{1}=1.516 \mu \mathrm{m}$ with $\sim 68 \%$ extinction and $\lambda_{2}=1.56 \mu \mathrm{m}$ with $\sim 99 \%$ extinction on resonance, with the measured loaded $Q_{l}$ factors $Q_{1}=1.8 \times 10^{4}$ and $Q_{2}=1.4 \times 10^{4}$, respectively. These quality factors are fairly representative for the fiber resonators with the best value obtained in (A) of $Q_{l} \sim 4 \times 10^{4}$ and the lowest in $(\mathrm{C})$ of $Q_{l} \sim 4 \times 10^{3}$. To demonstrate the thermal nonlinearity which manifests as a red shift of the resonant wavelength, a set of power dependent experiments were undertaken by scanning over the resonance at $1.56 \mu \mathrm{m}$, as shown in Fig. 1(b). This data reveals a linear dependence of the shift as a function power, with a relatively high coefficient of $4.5 \mathrm{pm} / \mu \mathrm{W}$ compared to on-chip resonators, which suggests that these fiber devices should be suitable for ultra low power operation.

Based on this observation, we set up a pump-probe experiment to exploit the thermooptic effect for low power all-optical amplitude modulation. A schematic of the experimental setup is shown in Fig. 2. Two continuous wave (CW) tunable external cavity lasers with 
a linewidth of $\sim 400 \mathrm{kHz}$ (Tunics Plus) are tuned to the WGM resonances $\lambda_{1}$ (pump) and $\lambda_{2}$ (probe), as shown in Fig. 1(a), and launched into the tapered fiber. The pump was modulated by a square wave using a pulse generator up to a frequency of $500 \mathrm{kHz}$. When the pulses enter the resonator they induce a thermal refractive index change which shifts the WGM resonant frequency with respect to the probe wavelength. When the energy in the resonator is sufficient to shift $\lambda_{2}$ by half the linewidth, the probe is no longer at resonance, resulting in its modulation. A wavelength tunable filter was used at the output to isolate the probe, which was monitored on an oscilloscope. For the experiments conducted in fiber (A), we found that a coupled pump power of only $\sim 10 \mu \mathrm{W}$ was required in the resonator to modulate the probe beam. Fig. 3(a) shows the input pump and (b) the output probe signal measured by the oscilloscope, at a frequency of $25 \mathrm{~Hz}$. At higher frequencies, for example $21 \mathrm{kHz}$ shown in Fig. 3(c), the output signal can still follow the modulation of the pump but becomes clipped.

To determine the thermal response time of the fiber resonators, Fig. 4(a) shows measurements of the peak-to-peak amplitude $\left(\mathrm{V}_{p-p}\right)$ of the probe beam versus the modulation frequency. At lower frequencies a flat response is observed, indicating that the thermal response time is faster than the modulation speed. However, when the modulation is faster than the response, a roll-off in the amplitude is observed, as seen via a comparison of Fig. 3(b) and (c). From the behavior shown in Fig. 4(a), a $-3 \mathrm{~dB}$ corner frequency of $21 \mathrm{kHz}$ can be measured. Using the usual bandwidth convention, the thermal response time of fiber (A) can be estimated as $\tau_{\theta}=1 /\left(2 \pi f_{-3 d B}\right)=7.5 \mu \mathrm{s}^{11}$. Despite differences in the a-Si:H material quality of the three fiber resonators, similar values of $\tau_{\theta}$ were obtained for fibers (B) $\tau_{\theta}=7 \mu \mathrm{s}$ and $(\mathrm{C}) \tau_{\theta}=7.2 \mu \mathrm{s}$, indicating that the response time is largely determined by the resonator size and shape.

Previous investigations of silicon resonators have shown that the nonlinear thermal properties can be used to infer the linear absorption coefficient of the material $\alpha^{12,13}$. These methods make use of the thermally induced resonance wavelength shift to provide a sensitive measure of the internal cavity absorption. Based on the approach in Ref. 12, we use the response time $\tau_{\theta}$ to calculate the absorbed power in the cavity $P_{A}$ then relate this to the corresponding intracavity energy $U_{c}$. To obtain $P_{A}$, we solve the thermal diffusion equation 
using a Laplace transform ${ }^{14}$ :

$$
\frac{d \Delta T}{d t}+\frac{\Delta T}{\tau_{\theta}}=\frac{P_{A}}{\rho C V}
$$

where $\rho=2.3 \times 10^{-3} \mathrm{~kg} / \mathrm{cm}^{3}$ is the material density of silicon, $C=705 \mathrm{~J} /(\mathrm{kgK})$ is the specific heat capacity, $V$ is the volume of the cavity, and $\Delta T$ is the temperature change. Here, $\Delta T$ is a function of the resonance shift $\Delta \lambda: \Delta T=\left(n_{c} \frac{\Delta \lambda}{\lambda_{0}}\right) /\left(\frac{d n}{d T}\right)^{12,15}$, where $n_{c}$ is the cavity refractive index, assumed to be 3.6 for a-Si:H at the "cold" (or low power) resonant wavelength $\lambda_{0}$, and the thermo-optic coefficient of a-Si:H is $d n / d T \sim 2.3 \times 10^{-4} \mathrm{~K}^{-1}$ Ref. 16 . Under the assumption that the temperature is time independent, the steady state solution of Eq. (1) yields:

$$
P_{A}=\frac{\rho C V \Delta T}{\tau_{\theta}} .
$$

To calculate $U_{c}$, we start from the equation which identifies the loss mechanisms that contribute to total (loaded) quality factor:

$$
Q_{l}^{-1}=Q_{e}^{-1}+Q_{i}^{-1}
$$

where $Q_{e}$ is the extrinsic (coupling) quality factor and $Q_{i}$ is the intrinsic quality factor. Here, $Q_{i}$ includes contributions from the losses associated with the resonator photon lifetime, the surface scattering, the bulk material, some leakage due to the lack of lateral confinement, and other parasitic losses of the system. However, we have previously shown in Ref. 6 that the losses associated with the radiation and surface scattering are negligible in these fiber resonators. As our system is in the overcoupled regime, $Q_{e}$ can be found from low power measurements of $Q_{l}$ using the relation ${ }^{12} Q_{e}=2 Q_{l} /\left(1+\sqrt{T_{\min }}\right)$, where $T_{\min }$ is the fraction of transmitted optical power measured by the photodetector at $\lambda_{0}$. Eq. (3) can then be used to calculate $Q_{i}$ for all input powers, and subsequently the cavity energy through ${ }^{12}$ : $U_{c}=Q_{i} P_{d} / \omega$, where $P_{d}$ is the power dropped to the resonator, and $\omega$ is the optical frequency.

Following the method outlined by Borselli et $a .^{12}$, in Fig. 4(b) we plot $P_{A}$ as a function of $U_{c}$ for the power dependent measurements of fiber (A) shown in Fig. 1(b). For the power levels used in our experiments, the absorbed power scales linearly with the intracavity energy as $P_{A} \sim \gamma_{l i n} U_{c}$. Using the fitted coefficient $\gamma_{l i n}$, it is then possible to estimate a value for the linear material absorption $\alpha$ via:

$$
Q_{\text {mat }}=\frac{\omega}{\gamma_{\text {lin }}}=\frac{2 \pi n_{c}}{\alpha \lambda_{0}}
$$


Applying this analysis for each of the fibers, we obtain the associated loss values of: (A) $\sim 3.4 \mathrm{~dB} / \mathrm{cm},(\mathrm{B}) \sim 8 \mathrm{~dB} / \mathrm{cm}$ and $(\mathrm{C}) \sim 17 \mathrm{~dB} / \mathrm{cm}$, which are all systematically slightly larger than the losses from the cutback measurements. We attribute the discrepancy between these results to the fact that the loss values established via the two methods correspond to slightly different wavelengths, with the losses from the cutback at $1.55 \mu \mathrm{m}$ and those from the cavity absorption at the resonant wavelength of $1.516 \mu \mathrm{m}$. Wavelength dependent measurements of the losses in a-Si:H fibers have previously revealed that typical values at $1.51 \mu \mathrm{m}$ are $\sim 1 \mathrm{~dB} / \mathrm{cm}$ higher than those at $1.55 \mu \mathrm{m}^{10}$, and when this difference is taken into consideration, the losses obtained via the two methods are brought into close agreement.

In conclusion, the thermal nonlinearity in atomically smooth, high quality a-Si:H fiber based microresonators have been characterized through a simple all-optical amplitude modulation experiment. Using the thermal response time and the power dependent resonance wavelength shift it is possible to estimate the linear absorption coefficient of the resonator materials. This loss characterization method has the advantage that it is non-destructive and can potentially find application in spherical or even bottle shaped WGM resonators, where cutback measurements cannot be applied.

The authors acknowledge EPSRC (EP/G051755/1 and EP/G028273/1), NSF (DMR0806860) and the Penn State Materials Research Science and Engineering Center (NSF DMR-0820404) for financial support. ACP holds a Royal Academy of Engineering fellowship. 


\section{REFERENCES}

${ }^{1}$ L. Yang, D. K. Armani, and K. J. Vahala, Appl. Phys. Lett. 83, 825 (2003).

${ }^{2}$ M. A. Foster, J. S. Levy, O. Kuzucu, K. Saha, M. Lipson, and A. L. Gaeta, Opt. Express 19, $14233(2011)$.

${ }^{3}$ V. R. Almeida and M. Lipson, Opt. Lett. 29, 2387 (2004).

${ }^{4}$ V.B. Braginsky, M. L. Gordetsky, and V. S. Ilchenko, Phys. Lett. A 137, 393 (1989).

${ }^{5}$ V.S. Ilchenko and M. L. Gorodetskii, Laser Phys. 2, 1004 (1992).

${ }^{6}$ N. Vukovic, N. Healy, P. Horak, J. R. Sparks, P. J. A. Sazio, J. V. Badding, and A. C. Peacock, Appl. Phys. Lett. 99, 031117 (2011).

${ }^{7}$ N. Healy, L. Lagonigro, J. R. Sparks, S. Boden, P. J. A. Sazio, J. V. Badding, and A. C. Peacock, Opt. Lett. 36, 2480 (2011).

${ }^{8}$ P. Mehta, M. Krishnamurthi, N. Healy, N. F. Baril, J. R. Sparks, P. J. A. Sazio, V. Gopalan, J. V. Badding, and A. C. Peacock, Appl. Phys. Lett. 97, 071117 (2010).

${ }^{9}$ P. Wang, T. Lee, M. Ding, A. Dhar, T. Hawkins, P. Foy, Y. Semenova, Q. Wu, J. Sahu, G. Farrel, J. Ballato, and G. Brambilla, Opt. Lett. 37, 728 (2012).

${ }^{10}$ L. Lagonigro, N. Healy, J. R. Sparks, N. F. Baril, P. J. A. Sazio, J. V. Badding, and A. C. Peacock, Appl. Phys. Lett. 96, 041105 (2010).

${ }^{11}$ H. Rokhsari and K. Vahala, Opt. Lett. 30, 427 (2005).

${ }^{12}$ M. Borselli, T. J. Johnson, and O. Painter, Opt. Express 13, 1515 (2005).

${ }^{13}$ P. E. Barclay, K. Srinivasan, and O. Painter, Opt. Express 13, 801 (2005).

${ }^{14}$ A. E. Fomin, M. L. Gorodetsky, I. S. Grudinin, and V. S. Ilchenko, J. Opt. Soc. Am. B 22, 459 (2005).

${ }^{15}$ C. A. Barrios, V. R. Almeida, R. R. Panepucci, B. S. Schmidt, and M. Lipson, IEEE Photon. Technol. Lett. 16, 506 (2004).

${ }^{16}$ F. G. Della Corte, M. E. Montefusco, L. Moretti, I. Rendina, and A. Rubino, Appl. Phys. Lett. 79, 168 (2001). 
FIG. 1. (a) Transmission spectrum (fiber A). Inset: Lorentzian fit (dashed line) to determine $Q$ for $1.56 \mu \mathrm{m}$ resonance, at low $(\sim 14 \mu \mathrm{W})$ power. (b) Transmission spectrum as a function of coupled input power as labeled in legend.

FIG. 2. Experimental setup for all-optical modulation.

FIG. 3. Modulated (a) pump and (b) probe signals at $25 \mathrm{~Hz}$. (c) Modulated probe at $21 \mathrm{kHz}$.

FIG. 4. (a) Measured amplitude response of the probe beam as a function of the modulation frequency of the pump. The dashed lines show a $-3 \mathrm{~dB}$ corner frequency of $21 \mathrm{kHz}$. (b) Absorbed power versus cavity energy. Results obtained for fiber (A). 